\title{
Effective rocking motion for inducing sleep in adults - Verification of effect of mother's embrace and rocking motion -
}

\author{
Keishi Ashida ${ }^{* * *}$, Yoshifumi Morita ${ }^{* * *}$, Ryojun Ikeura ${ }^{* * * * * *}$, Kiyoko Yokoyama*******, Ming Ding **, Yuki Mori** \\ *Department of Computer Science and Engineering, Nagoya Institute of Technology \\ Gokiso, Syouwa, Nagoya, Aichi 466-8555, Japan \\ E-mail: k.ashida.353@stn.nitech.ac.jp / morita@nitech.ac.jp \\ **RIKEN- SUMITOMORIKO Collaboration Center for Human-Interactive Robot Research (RSC RIKEN) \\ 2271-130 Anagahora, Shimoshidami, Moriyama, Nagoya, Aichi 463-0003, Japan \\ ***Department of Mechanical Engineering, Mie University \\ 1577 Kurimamachiyacho, Tsu, Mie 514-8507, Japan \\ ****Department of Design and Architecture, Nagoya City University \\ 2-1-10 Kitachikusa, Chikusa, Nagoya, Aichi 463-0083, Japan
}

\begin{abstract}
We investigated the most effective rocking motion for inducing sleep in adults. We prepared ten types of rocking motions, including two types of mother's rocking motions. The sleep-inducing effect of all the rocking motions was evaluated using Thurstone's paired comparison method (Case V). From statistical analysis of the subjective experimental results we found that, of the ten types of rocking motions, the linear motion component of a mother's rocking motions (Type 2) was the most effective for inducing sleep in adults.
\end{abstract}

Keywords: Rocking motion, Mother's rocking motion, Sleep-inducing effect, Thurstone's paired comparison method

\section{Introduction}

Recently, the number of people suffering from high levels of stress has increased, a phenomenon that has been called a stressful society. ${ }^{1}$ Every year, more and more people suffer from stress-related illnesses. Moreover, sleep disorders are becoming more widespread in modern society because stress is one of the causes of insomnia. In nursing homes, it is a large burden for caregivers to assist patients to ensure the wellbeing of people who suffer from these problems. ${ }^{2}$ Therefore, stress reduction and sleep induction are necessary for many people in modern society. It is generally known that when a baby is embraced and rocked by his or her mother, the baby feels comfortable and falls asleep quickly. Therefore, we hypothesized that a rocking motion simulating a mother's embrace and rocking motion would have the same effects on adults. Against this background, the aim of our project is to develop a relaxation machine for reducing stress and inducing sleep by using a rocking motion simulating that of a mother. We expect that our research results will be used for other relaxation systems or robots in the future, for example the Robot for Interactive Body Assistance (RIBA), which is designed to assist caregivers by lifting patients in and out of their beds and wheelchairs. ${ }^{3}$

In our previous study, we analyzed a mother's embrace and rocking motion, and we designed and constructed an excitation apparatus simulating a mother's rocking motion. We found that there are two 
types of rocking motions given by a mother, and we constructed two models of these rocking motions. ${ }^{4}$ From analysis of brain signals and subjective assessment, we found that the mother's rocking motions were more effective for inducing sleep in two healthy adults than in the case without rocking. ${ }^{5}$ However, there were two problems with that study. One is that the number of subjects was small, and the other is that the sleepinducing effects of different rocking motions have to be investigated.

In the work described in this paper, in addition to the two types of mother's rocking motions mentioned above, we prepared eight types of rocking motions to find the rocking motion that is most effective for inducing sleep in adults. We performed three experiments and evaluated the sleep-inducing effect by using Thurstone's paired comparison method.

\section{Excitation apparatus simulating a mother's rocking motion}

In our previous work we analyzed a mother's embrace and rocking motion to identify the features of the motion. From the results, we modeled a mother's rocking motion. The model of a mother's rocking motion is represented by

$\left\{\begin{array}{l}x^{r e f}(t)=A_{x} \sin \left(2 \pi f_{x} t\right) \\ \theta^{\text {ef }}(t)=A_{\theta} \sin \left(2 \pi f_{\theta} t+\phi\right)+B_{\theta}\end{array}\right.$

where $x^{r e f}(t)$ and $\theta^{\text {ref }}(t)$ are the position and the angle of the center of gravity of the baby, respectively, and $B_{\theta}$ was determined from the angle of a comfortable sitting position reported in Ref. 6. Then, we realized this rocking motion using the excitation apparatus shown in Fig. 1. The excitation apparatus has two degrees of freedom, one for linear motion and one for rotational motion. The rocking chair is driven by a linear motor and a rotary motor. The rotary motor is connected to the chair through a timing belt for rotation. The drive systems of the excitation apparatus are composed of speed-reference-type servo amplifiers for the linear and rotary motors, linear and rotary encoders, and a DSP board (dSPACE DS1104). The DSP board is used to implement the controllers for the linear and rotary motors. We designed controllers for the excitation apparatus using PD control and feed-forward control in

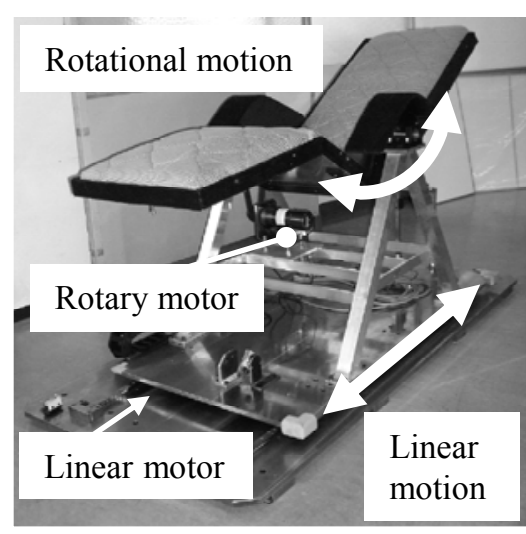

Fig. 1. Photograph of excitation apparatus.

order to simulate a mother's rocking motions in the excitation apparatus.

\section{Experimental method}

We performed experiments for finding the mosteffective rocking motion for inducing sleep. The sleepinducing effect of each rocking motion was evaluated on a numerical scale using Thurstone's paired comparison method (Case V) ${ }^{7,8}$. We verified the validity of adopting this method by using Mosteller's goodnessof-fit test. Moreover, we evaluated the confidence of the scale values.

The subjects were nine healthy males (average age, 25.4 years). The subjects wore ear muffs for sound insulation during the experiment, had their eyes closed, and rested in a supine position on the chair which was rocked by the excitation apparatus. The experimental protocol is shown in Fig. 2. R1, R2, R3, and R4 denote the types of rocking motions used in the experiment. After every two rocking motions, the subjects answered the question, "Which of the two rocking motions was more effective for inducing sleep?". The evaluation was performed with six different combinations of four rocking motions for each experiment. In order to reduce the influence of an order effect, we divided the subjects into two groups and used six combinations (Types R1and R2, Types R1 and R3, Types R1 and R4, Types R2 and R3, Types R2 and R4, and Types R3 and R4) in two different orders, namely, Procedure 1 and Procedure 2, as shown in Fig. 2. 


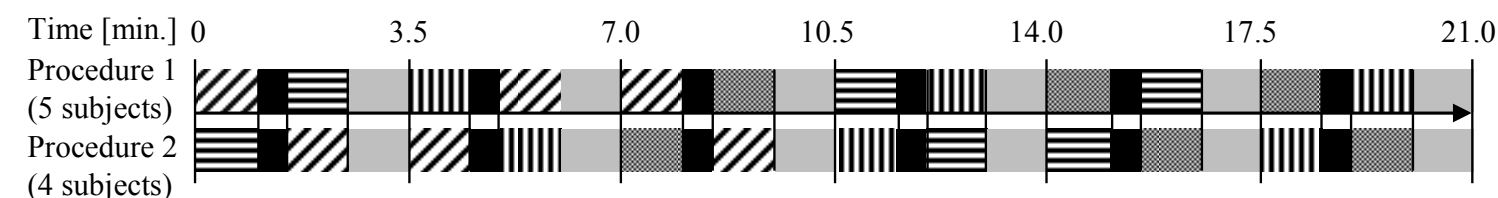

Eye closed and relaxed with the rocking vibration

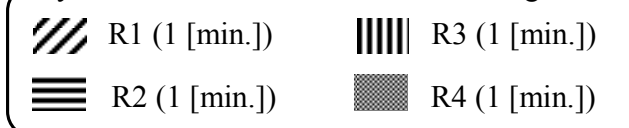

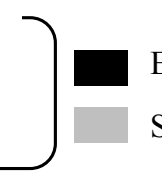

Eye closed and relaxed without rocking (0.5 [min.])

Subjective assessment (1 [min.])

Fig. 2. Experimental protocol for finding the most-effective rocking motion in Experiments I, II, and III.

\section{Experiments}

We performed three different experiments, Experiments I, II, and III, which are detailed below.

\subsection{Rocking motions used in Experiment I}

We prepared four types of rocking motions, namely two types of mother's rocking motions (Type 1 and Type 2), a rocking motion imitating the motion of a rocking chair (Type 3), and a rocking motion that has a high correlation with motion sickness (Type 4). In Fig. 2, R1, R2, R3, and R4 correspond to Type 1, Type 2, Type 3, and Type 4 , respectively. Type 3 was added to the candidates because a rocking chair is known to have a relaxation effect. ${ }^{9}$ Type 4 was added to the candidates as an uncomfortable rocking motion in this comparative study. The parameters of Type 1, Type 2, and Type 4 are shown in Table 1.

In order to produce the motion of a rocking chair shown in Fig. 3, we first measured the motion of an actual rocking chair by using a $3 \mathrm{D}$ motion capture system (Motion Analysis) when an adult male was seated in the rocking chair. Type 3 is the rocking motion produced after the experimenter lowered the backrest of the rocking chair and released it. The motion of the rocking chair consists only of rotary motion. We set the amplitude of the rotary motion to as large a value as possible within a range that was comfortable for the subject. Moreover, the rotary angle in the steady state was set to 25 degrees, which is the same as the angle determined from the angle of a comfortable sitting position reported in Ref. 6. The Type 3 rocking motion is shown in Fig. 4.

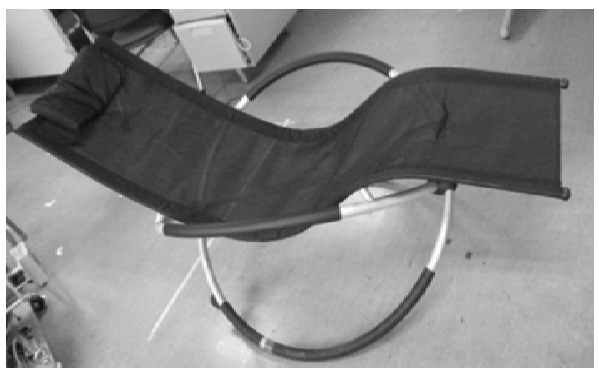

Fig. 3. Photograph of rocking chair.

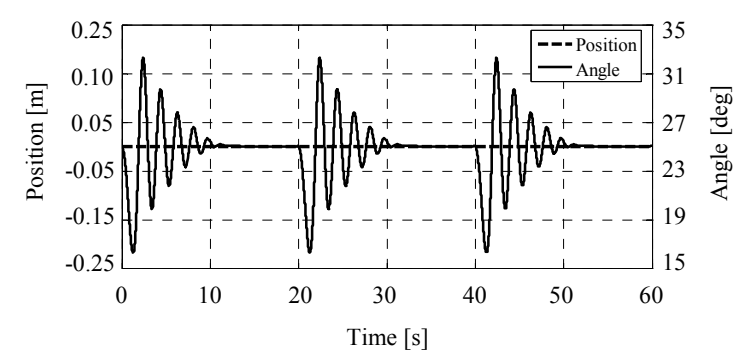

Fig. 4. Rocking motion (Type 3).

The Type 4 rocking motion is represented by Eq. (1). The frequency was determined to be $0.063 \mathrm{~Hz}$, which has been shown to have a high correlation with motion sickness ${ }^{10}$. The amplitude was determined so that the acceleration was almost the same as those of other rocking motions and within the range of acceleration that can be produced by the excitation 
Table 1. Parameters of the rocking motions.

\begin{tabular}{cccccccccc}
\hline Parameter & Type 1 & Type 2 & Type 4 & Type 5 & Type 6 & Type 7 & Type 8 & Type 9 & Type 10 \\
\hline$A_{x}[\mathrm{~m}]$ & 0.115 & 0.098 & 0.250 & 0.095 & 0.100 & 0.098 & 0.098 & 0.060 & 0 \\
$A_{\theta}[\mathrm{deg}]$ & 2.455 & 2.734 & 10.0 & 3.136 & 2.332 & 2.734 & 0 & 0 & 2.734 \\
$B_{\theta}[\mathrm{deg}]$ & 25 & 25 & 25 & 25 & 25 & 25 & 25 & 25 & 25 \\
$f_{x}[\mathrm{~Hz}]$ & 0.326 & 0.234 & 0.063 & 0.226 & 0.243 & 0.234 & 0.234 & 0.468 & 0 \\
$f_{\theta}[\mathrm{Hz}]$ & 0.326 & $0.234 \times 2$ & 0.063 & $0.226 \times 2$ & $0.243 \times 2$ & $0.234 \times 2$ & 0 & 0 & $0.234 \times 2$ \\
$\phi[\mathrm{rad}]$ & $\pi$ & $\pi / 2$ & $\pi$ & $\pi / 2$ & $\pi / 2$ & $-\pi / 2$ & $\pi / 2$ & $\pi / 2$ & $\pi / 2$ \\
\hline
\end{tabular}

apparatus. The parameters of the Type 4 motion are also shown in Table 1.

\subsection{Results in Experiment I}

From the results of the paired comparison method, we obtained the frequency distribution matrix shown in Table 2. Table 2 shows the number of subjects who offered the response "The first rocking motion was more effective for inducing sleep than the second rocking motion". The first and second rocking motions are shown in the rows and columns of Table 2, respectively. The scale values for the sleep-inducing effect of the rocking motions were derived by applying Thurstone's paired comparison method (Case V) to Table 2. The scale values are shown in Table 3. From Table 3, we can rank the four types of rocking motions based on their sleep-inducing effects as follows:

\section{Type $2>$ Type $1>$ Type $3>$ Type 4 .}

Moreover, we verified the validity of adopting Thurstone's paired comparison method (Case V) by using Mosteller's goodness-of-fit test. The goodness-offit, $\chi^{2}$, was 3.52 , and the flexibility, $d f$, was 3 , meaning that a $5 \%$ significance level was not rejected. Therefore, Thurstone's paired comparison method (Case V) can be adopted for evaluating the sleep-inducing effect.

We examined the significant differences between the four rocking motions by using the method proposed in Ref. 11. The result is shown in Fig. 5. We found that the $95 \%$ confidence interval of Type 1 overlapped with that of Type 3 . The combination of Type 1 and Type 3 showed no significant difference, whereas the other five combinations showed significant differences. In other words, of the four types of rocking motions, we found that Type 2 was the most effective for inducing sleep in adults and Type 4 was the least effective.

Table 2. Frequency distribution matrix in Experiment I.

\begin{tabular}{c|cccc}
\hline & Type 1 & Type 2 & Type 3 & Type 4 \\
\hline Type 1 & - & 6 & 3 & 2 \\
Type 2 & 3 & - & 2 & 0 \\
Type 3 & 6 & 7 & - & 2 \\
Type 4 & 7 & 9 & 7 & - \\
\hline
\end{tabular}

Table 3. Scale values of different rocking motions in Experiment I.

\begin{tabular}{c|cccc}
\hline & Type 1 & Type 2 & Type 3 & Type 4 \\
\hline Scale value & 0.19 & 1.06 & -0.11 & -1.15 \\
\hline
\end{tabular}

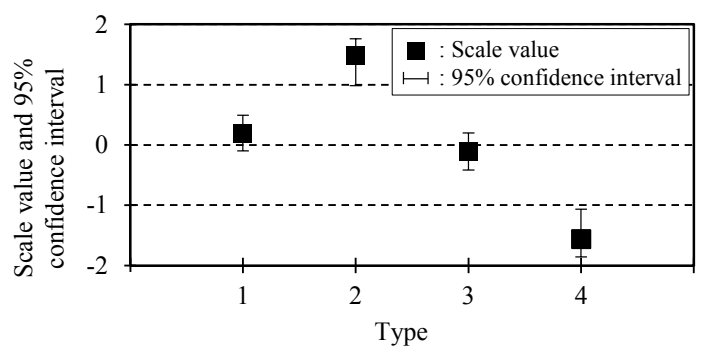

Fig. 5. Scale values and $95 \%$ confidence intervals of the four types of rocking motions in Experiment I.

\subsection{Rocking motions used in experiment II}

We prepared three types of rocking motions, namely Type 5, Type 6 and Type 7, in addition to Type 2. In Fig. 2, R1, R2, R3, and R4 correspond to Type 2, Type 5 , Type 6, and Type 7, respectively. Type 5 was a rocking motion based on one given to a $5 \mathrm{~kg}$ baby in another study ${ }^{5}$, and Type 6 was a rocking motion based 
on one given to a $7 \mathrm{~kg}$ baby in the same study. More details of these rocking motions are shown in Ref. 5 . Type 7 was the same as Type 2 except for the phase, which differed by $180 \mathrm{deg}$. These parameters are shown in Table 4.

\subsection{Results in Experiment II}

Figure 6 shows the scale values and the $95 \%$ confidence intervals for the sleep-inducing effect of the rocking motions obtained using Thurstone's paired comparison method (Case V) and the method proposed in Ref. 11. From the scale values in Fig. 6, we can rank the four types of rocking motions based on their sleep-inducing effects as follows:

\section{Type $2>$ Type $6>$ Type $5>$ Type 7 .}

Moreover, the validity of adopting Thurstone's paired comparison method (Case V) for evaluating the sleepinducing effects was confirmed in the same way as in Experiment I.

In Fig. 6, the combination of Type 2 and Type 7 showed a significant difference, whereas the other five combinations showed no significant differences. We concluded that, of the four types of rocking motions, Type 2 was the most effective for inducing sleep in adults. As for the reason why no significant differences appeared, we considered that the evaluations were uneven because it was difficult for subjects to discriminate the small differences in the frequency and amplitude. In this situation, Type 7 was chosen as the least effective rocking motion. With the Type 7 motion, the subjects reported slight motion sickness.

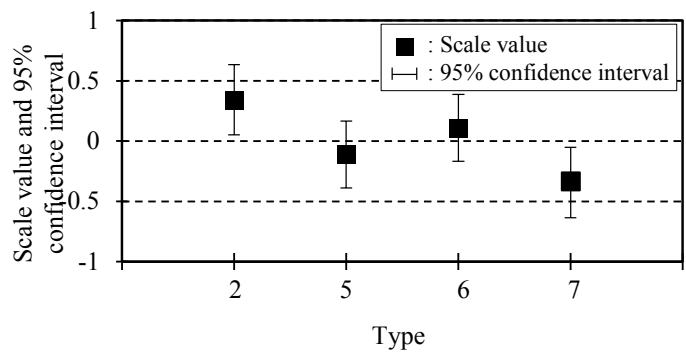

Fig. 6. Scale values and $95 \%$ confidence intervals of the four types of rocking motions in Experiment II.

\subsection{Rocking motions used in Experiment III}

In order to find a more effective rocking motion for inducing sleep, in Experiment III, we focused on the components of the Type 2 motion. We prepared three types of rocking motions, namely, Type 8, Type 9 and Type 10, in addition to Type 2. In Fig. 2, R1, R2, R3, and R4 correspond to Type 2, Type 8, Type 9, and Type 10 , respectively. Type 8 was a rocking motion consisting of only the linear motion component of Type 2. Type 9 was also a rocking motion consisting of only linear motion that was reported to be effective for inducing sleep in babies in Ref. 12. Type 10 was a rocking motion consisting of only the rotational motion component of Type 2 .

When the frequency of the rocking motion used in Ref. 12 is used instead of the frequency of Type 8, the acceleration is too large. For this reason, the amplitude $A_{x}$ was set to a smaller value, and the frequency $f_{x}$ was set to a small value, namely, the frequency $f_{\theta}$ of the rotary motion component of Type 7. This rocking motion is Type 9. These parameters are shown in Table 1.

\subsection{Results in Experiment III}

Figure 7 shows the scale values and the $95 \%$ confidence intervals for the sleep-inducing effect of the rocking motions obtained by using Thurstone's paired comparison method (Case V) and the method proposed in Ref. 11. From the scale values in Fig. 7, we can rank the four types of rocking motions based on their sleepinducing effects as follows:

$$
\text { Type } 8>\text { Type } 10>\text { Type } 2>\text { Type } 9 \text {. }
$$

Moreover, the validity of adopting Thurstone's paired comparison method (Case V) for evaluating the sleepinducing effect was confirmed in the same way as in Experiment I.

In Fig. 7, the three combinations including Type 9 (Types 2 and 9, Types 8 and 9, and Types 9 and 10) showed significant differences, whereas the other three combinations showed no significant differences. However, there is a significant difference in the combination of Type 8 and Type 10 if the confidence interval is changed to $80 \%$ instead of $95 \%$. Therefore, of the four types of rocking motions, we concluded that 
Type 8 is the most effective for inducing sleep in adults. The reason why Type 9 was identified as the least effective rocking motion was that the acceleration of Type 9 is too large, based on the subjects' opinions. We found that the acceleration was related to the effective motion for inducing sleep.

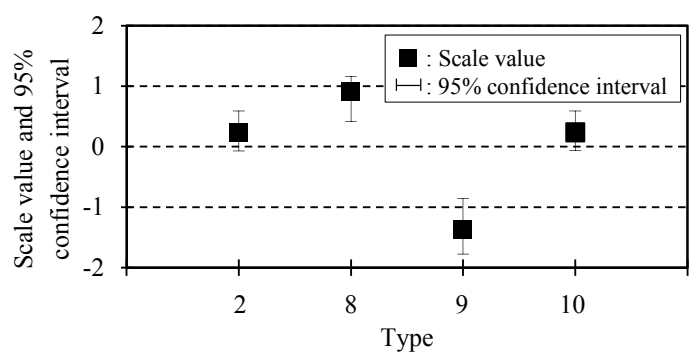

Fig. 7. Scale values and $95 \%$ confidence intervals of the four types of rocking motions in Experiment III.

\section{Conclusion}

From subjective experimental results, we found that, of the ten types of rocking motions examined, the linear motion component of a mother's rocking motion (Type 2) was the most effective rocking motion for inducing sleep in adults.

In future work, we plan to confirm that the Type 8 rocking motion, which is the linear motion component of a mother's rocking motion (Type 2), is effective for inducing sleep and reducing stress in adults by using electroencephalogram (EGG) analysis. We will also compare the Type 8 rocking motion and aromatherapy, which is a popular natural way of inducing sleep and bringing about relaxation.

\section{References}

1. Ito, H. and Ishino, Y., Adverse Effects of Sleep Deprivation, Iho Fuji, No.124 (2003) (in Japanese), p.1.

2. Mishima, K., Sleep Problems in Dementia, Proceedings of the Annual Meeting of the Japanese Research Group on Senile Dementia, Vol.17 (2010) (in Japanese), pp.109113.
3. RIKEN-TRI Collaboration Center for Human-Interactive Robot Research (RTC), World's first robot that can lift up a human in its arms, RIBA, (online), available from $<$ http://rtc.nagoya.riken.jp/RIBA/index-e.html>, (accessed on 13 June, 2014)

4. Yamaguchi, K., Kobayashi, H., Morita, Y. Sakaida, Y. Ikeura, R. and Yokoyama, K., Motion Analysis of Mother's Embracing and Rocking a Baby and Development of Excitation Apparatus, Proc. of Int. Conf. on Control, Automation and Systems 2011 (ICCAS2011) (2011) pp.1792-1796.

5. Morita, Y., Yamaguchi, K., Ashida, K., Ikeura, R. and Yokoyama, K., Verification of Sleep-Inducing Effect by Excitation Apparatus Simulating Mother's Embrace and Rocking Motion, Proceedings of the 9th International Workshop on Robot Motion and Control (RoMoCo '13) (2013) , pp.80-85.

6. Sasaki, Y., Kawamoto, T. and Ymazaki, N., Development of an Experiment Bed for Searching Comfort Semi-Sitting Position, Japanese Journal of Ergonomics, Vol.42, No.6 (2006), pp.373-380.

7. Research Committee of Sensory Evaluation, Sensory Evaluation Handbook, JUSE ed., JUSE Press, Ltd., Japan (1973), pp.471-476.

8. Ichikawa, H., A Study of Representing Content for a Special 3D Display (2010) (in Japanese), pp.34-41.

9. Tamakoshi, S., Yamamoto, N., Morimoto, F., Nagae, S., Ichiba, H. and ISO, H., Engineering Psychology on the Relaxation Effects of a Rocking chair, Behavioral Science Research, Vol.50, No.2 (2012) (in Japanese), pp.101-105.

10. Siroto, H., Evaluation Method of Motion Sickness in Trains Using Low-frequency Lateral Vibration, Railway Research Review, Vol.130 (2006) (in Japanese), pp.3839.

11. Tabata, Y., Ohga, Y., Kakuta, M., Nakamae, M., Morioka, M., Uto, F., Okunishi, T., Oti, T. and Maeda, K., Confidence Coefficient of Subjective Scale Value in Method of Paired Comparisons (Case V), Japanese Society of Radiological Technology, Vol.51, No.4 (1995) (in Japanese), pp.445-449.

12. Fuji, T., Furusawa, T., Takenouchi, T. and Tomotari, M., Development of Electric Bed for Babies for Parenting Support, Japan Society for Design Engineering (2006) (in Japanese), pp.19-20. 